\title{
Contributo alla conoscenza \\ delle caratteristiche sismiche dell'Iran settentrionale
}

\author{
F. Peronaci
}

ricevuto il 5 novembre 1959

In una precedente nota abbiamo accennato alle carateristiche sismiche dell'Altipiano Iraniano con particolare riguardo alla sua sismicità, ed iniziato lo studio del forte terremoto del 13 dicembre 1957 del quale abbiamo determinato le coordinate ipocentrali e il tempo origine. Nel presente lavoro ci siamo preoccupati di determinare la natura fisica del movimento tellurico all'ipocentro, e abbiamo iniziato lo studio di un altro terremoto avvenuto nello stesso anno a Nord di Teheran nel massiccio del Demavend.

NATURA FISICA DELLA SCOSSA ALL'IPOCENTRO DEL TERREMOTO DEL 13 DICEMRRE 1957.

Ricordiamo che la possibilità di indagare sulla natura fisica della causa che ha prodotto un movimento sismico, è legata all'esame degli impulsi iniziali registrari in un certo numero di stazioni distribuite secondo tutte le possibili direzioni intorno all'epicentro; partendo da questi dati sono state sviluppate varie teorie che hanno fornito risultati del massimo interesse circa il tipo di forza che origina un terremoto.

Notevole a tale riguardo è la teoria sviluppata da Byerly per terremoti superficiali basata sulla ipotesi di una Terra omogenea, e quindi che implica la sostituzione della traiettoria curvilinea del raggio sismico con la rettilinea, e successivamente estesa da Di Filippo ai terremoti profondi. Il metodo è molto noto per cui ci limiteremo a riportare i risultati da noi ottenuti applicandolo ai due terremoti su accennati. Partiti dalle coordinate ipocentrali da noi ricavate analiticamente per il terremoto del 13 Dicembre 1957

$$
\begin{aligned}
& \varphi=34^{\circ} 36^{\prime} 53^{\prime \prime} \quad N \pm 0^{\circ} 0^{\prime} 47^{\prime \prime} \\
& \lambda=47^{\circ} 48^{\prime} 43^{\prime \prime} \quad E \pm 0^{\circ} 0^{\prime} 52^{\prime \prime} \\
& h=7 \pm 5,3 \mathrm{~km}
\end{aligned}
$$


abbiamo determinato la distanza epicentrale $\Delta$ e l'azimut $a$ rispetto all'epicentro, di ognuna delle 65 stazioni a nostra disposizione, elementi necessari per passare alla rappresentazione cartografica, che nel nostro caso è la stereografica polare con epicentro coincidente con l'ipocentro, polo di proiezione l'antiepicentro, e piano di proiezione il piano equatoriale cor rispondente.

Successivamente abbiamo calcolato la distanza epicentrale $\Delta^{\prime}$ corrispondente al tragitto rettilineo del raggio sismico, dato che 11 modello di Terra a cui si appllca il metodo è supposto omogeneo, e siamo perrenuti (Tabella I) agli elementi che consentono di riportare in proiezione stereografica le posizioni delle varie stazioni rispetto all'epicentro. Come è noto, per il calcolo di $\Delta^{\prime}$ è necessario appllcare la relazione di Benndorf.

$$
\cos e=\frac{V_{o}}{v_{n}}
$$

ove $V_{0}$ è la velocità all'ipocentro, $v_{n}$ la velocità apparente del raggio sismico in corrispondenza della distanza epicentrale relativa alla stazione considerata. A questo riguardo le $v_{n}$ sono state determinate dalle dromocrone di Jeffreys per terremoti con ipocentri superficiali mentre per $V_{o}$ si è assunto $l 1$ valore di $7,77 \mathrm{Km} / \mathrm{sec}$. dato che i calcoll sono stati riferiti alla Terra privata della crosta terrestre e gll mpulsi studiati si riferiscono alle onde $P$.

Tabella I

\begin{tabular}{|c|c|c|c|c|c|c|}
\hline Stazione & 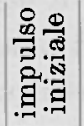 & $\begin{array}{c}\Delta \\
(\mathrm{km})\end{array}$ & $\begin{array}{c}a \\
\text { (gradi) }\end{array}$ & $\begin{array}{c}v_{n} \\
(\mathrm{~km} / \mathrm{sec})\end{array}$ & $\cos e$ & $\operatorname{tang} \frac{1}{2} \Delta^{\prime}$ \\
\hline $\begin{aligned} 1 & \text { Goris } \\
2 & \text { Nachichevan } \\
3 & \text { Kirovadan } \\
4 & \text { Stepanovan } \\
5 & \text { Bogdanova } \\
6 & \text { Tiflis } \\
7 & \text { Akhalkanati } \\
8 & \text { Abas-Tunian } \\
9 & \text { Zugdidi } \\
10 & \text { Aschkabad } \\
11 & \text { Erevan } \\
12 & \text { Ksara } \\
13 & \text { Piatigorsk } \\
14 & \text { Boriom } \\
15 & \text { Sochi } \\
16 & \text { Feodosia }\end{aligned}$ & $\begin{array}{l}+ \\
+ \\
+ \\
+ \\
+ \\
+ \\
+ \\
+ \\
+ \\
+ \\
+ \\
+ \\
+ \\
+\end{array}$ & $\begin{array}{r}532 \\
533 \\
671 \\
752 \\
798 \\
814 \\
824 \\
886 \\
939 \\
993 \\
1079 \\
1094 \\
1107 \\
1194 \\
1200 \\
1549\end{array}$ & $\begin{array}{r}346,1 \\
336,9 \\
349,5 \\
337,4 \\
334,1 \\
342,0 \\
333,9 \\
332,1 \\
328,3 \\
68,7 \\
345,7 \\
269,9 \\
339,8 \\
342,0 \\
326,9 \\
320,7\end{array}$ & $\begin{array}{l}7,81 \\
7,81 \\
7,87 \\
7,90 \\
7,91 \\
7,92 \\
7,92 \\
7,93 \\
7,94 \\
7,95 \\
7,96 \\
7,97 \\
8,04 \\
8,10 \\
8,10 \\
8,43\end{array}$ & $\begin{array}{r}0,99488 \\
99488 \\
98729 \\
98354 \\
98230 \\
98106 \\
98106 \\
97882 \\
97858 \\
97735 \\
97613 \\
97494 \\
96641 \\
95925 \\
95925 \\
92170\end{array}$ & $\begin{array}{l}0,102 \\
0,102 \\
0,161 \\
0,184 \\
0,191 \\
0,197 \\
0,197 \\
0,204 \\
0,210 \\
0,216 \\
0,222 \\
0,228 \\
0,266 \\
0,295 \\
0,295 \\
0,421\end{array}$ \\
\hline
\end{tabular}


Tabella I

\begin{tabular}{|c|c|c|c|c|c|c|c|}
\hline & Stazione & 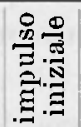 & $\begin{array}{c}\Delta \\
(\mathrm{km})\end{array}$ & $\begin{array}{c}a \\
\text { (gradi) }\end{array}$ & $\begin{array}{c}v_{n} \\
(\mathrm{~km} / \mathrm{sec})\end{array}$ & $\cos e$ & $\operatorname{tang} \frac{\frac{1}{2}}{2} \Delta^{\prime}$ \\
\hline 17 & Sinferopoli & + & 1622 & 318,2 & 8,47 & 91735 & 0,434 \\
\hline 18 & Elwan & + & 1630 & 255,9 & 8,47 & 91735 & 0,434 \\
\hline 19 & Samarcanda & - & 1777 & 66,8 & 8,64 & 89930 & 0,486 \\
\hline 20 & Istanbul & + & 1789 & 299,1 & 8,72 & 89105 & 0,509 \\
\hline 21 & Quetta & - & 1866 & 99,1 & 8,74 & 88901 & 0,515 \\
\hline 22 & Stalinabad & - & 1915 & 71,1 & 8,92 & 87107 & 0,564 \\
\hline $2 \overline{3}$ & Kuliab & - & 1994 & 73,1 & 8,95 & 86816 & 0,572 \\
\hline 24 & Teskhend & - & 2012 & 62,5 & 9,02 & 86141 & 0,589 \\
\hline 25 & Kirninev & + & 2085 & 316,2 & 9,02 & 86141 & 0,589 \\
\hline 26 & Karaki & - & 2140 & 114,8 & 9,63 & 80685 & 0,732 \\
\hline 27 & Bucarest & + & 2149 & 308,0 & 9,65 & 80518 & 0,736 \\
\hline 28 & Warsak & - & 2170 & 90,1 & 9,67 & 80351 & 0,741 \\
\hline 29 & Atene & + & 2183 & 286,2 & 9,68 & 80269 & 0,743 \\
\hline 30 & Jasi & + & 2189 & 315,8 & 9,68 & 80269 & 0,743 \\
\hline 31 & Namangan & - & 2198 & 64,3 & 9,70 & 80103 & 0,747 \\
\hline 32 & Andizhan & - & 2252 & 65,7 & 10,99 & 70700 & 1,000 \\
\hline $3 \overline{3}$ & Laore & - & 2495 & 93,2 & 11,21 & 69313 & 1,040 \\
\hline 34 & Sverdlosk & + & 2633 & 17,7 & 11,44 & 67919 & 1,081 \\
\hline 35 & Scalnate Pleso & + & 2774 & 314,2 & 11,68 & 66521 & 1,122 \\
\hline 36 & Derha Dun & - & 2833 & 92,3 & 11,80 & 65847 & 1,143 \\
\hline 37 & Varsavia & + & 2877 & 320,8 & 11,82 & 65736 & 1,146 \\
\hline 38 & Bratislava & + & 2940 & 310,8 & 12,06 & 64427 & 1,187 \\
\hline 39 & Vienna & + & 2998 & 309,8 & 12,19 & 63741 & 1,201 \\
\hline 40 & Bombay & & 3022 & 118,8 & 12,20 & 63688 & 1,210 \\
\hline 41 & Roma & + & 3165 & 295,3 & 12,32 & 63068 & 1,231 \\
\hline 42 & Trieste & + & 3170 & 301,2 & 12,33 & 63017 & 1,233 \\
\hline 43 & Praga & + & 3209 & 312,7 & 12,33 & 63017 & 1,233 \\
\hline 44 & Pavia & + & 3400 & 302,1 & 12,61 & 61617 & 1,278 \\
\hline 45 & Strasburgo & + & 3625 & 307,4 & 12,68 & 61277 & 1,289 \\
\hline 46 & Copenaghen & + & 3670 & 336,2 & 12,75 & 60941 & 1,330 \\
\hline 47 & Clermont & + & 3940 & 303,3 & 13,05 & 59540 & 1,349 \\
\hline 48 & Algeri & + & 4010 & 286,8 & 13,21 & 58819 & 1,375 \\
\hline 49 & Kiruna & + & 4085 & 343,4 & 13,21 & 58819 & 1,375 \\
\hline 50 & Kew & + & 4210 & 307,2 & 13,23 & 58730 & 1,378 \\
\hline 51 & Tamarraset & + & 4292 & 276,3 & 13,25 & 58641 & 1,381 \\
\hline 52 & Duram & + & 4500 & 310,4 & 13,50 & 57555 & 1,421 \\
\hline 53 & Astrida & + & 4542 & 208,3 & 13,53 & 57386 & 1,427 \\
\hline 54 & Lwiro & + & 4544 & 206,2 & 13,53 & 57386 & 1,427 \\
\hline 55 & Toledo & + & 4565 & 292,9 & 13,54 & 57385 & 1,427 \\
\hline 56 & Malaga & + & 4695 & 289,2 & 13,55 & 57343 & 1,428 \\
\hline 57 & Irkutsk & + & 4829 & 48,1 & 13,70 & 56715 & 1,452 \\
\hline 58 & Kiaka & + & 4968 & 51,0 & 13,72 & 56632 & 1,455 \\
\hline 59 & Kabansk & + & 4976 & 48,6 & 13,72 & 56632 & 1,455 \\
\hline 60 & Tananarive & & 5970 & 179,8 & 15,20 & 51118 & 1,681 \\
\hline 61 & Uglegorsk & + & 7463 & 45,2 & 17,90 & 43408 & 2,093 \\
\hline 62 & Iuzno. S. & + & 7613 & 47,1 & 18,04 & 43071 & 2,094 \\
\hline 63 & College & + & 8877 & 6,7 & 20,74 & 37463 & 2,475 \\
\hline 64 & Brébeuf & + & 9346 & 322,9 & 21,98 & 35350 & 2,646 \\
\hline 65 & Ottawa & 1 & 9464 & 323,9 & 21,99 & 35334 & 2,647 \\
\hline
\end{tabular}


Nella fig. 1 sono state riportate in proiezione stereografica la posizione delle 65 stazioni e il primo impulso è stato rappresentato con una freccia verso l'ipocentro se dilatazione e con una freccia diretta in senso contrario se compressione.

Esaminando la distribuzione ottenuta è facile tracciare con buona approssimazione i due cerchi normali previsti dalla teoria, passanti per

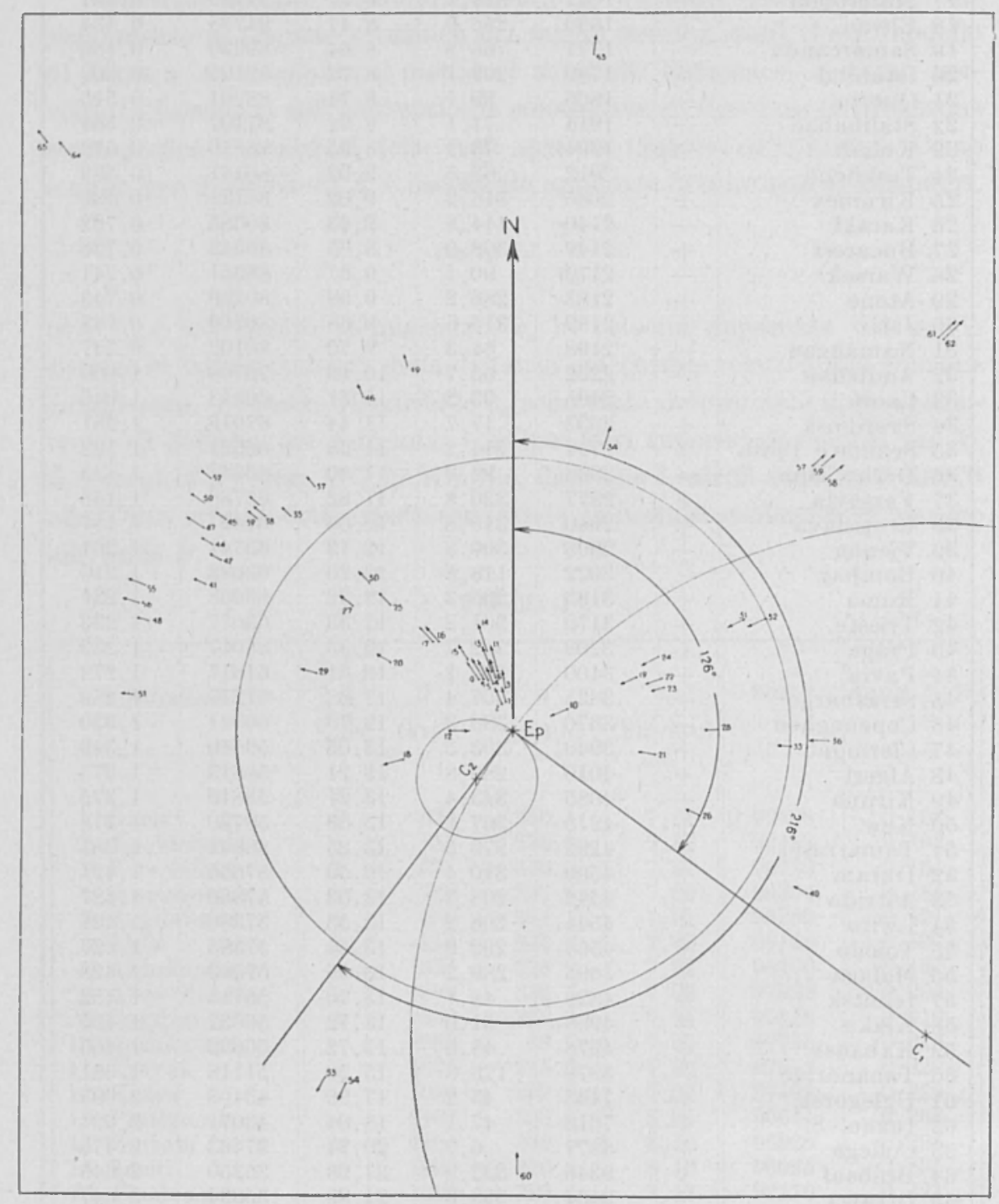

Fig. 1 
l'epicentro e che separano le compressioni dalle dilatazioni. Le due rette tangenti ai cerchi e passanti per l'epicentro ci forniscono le trace dei due possibili piani di frattura, ne è possibile come è noto, desumere dalla teoria quale dei due piani sia quello effettivo.

Il terremoto è quindi dovuto ad una frattura avvenuta secondo uno dei due piani formanti con il nord geografico gli angoli di $126^{\circ}$ e $216^{\circ}$ rispettivamente. Per quanto riguarda l'angolo che i piani formano rispetto al raggio terrestre per l'epicentro è sufficiente (fig. 2) tracciare un cerchio corrispondente alla sezione meridiana, riportare sulla traccia del piano equatoriale a partire dal centro i diametri dei due cerchi e unire l'estremo dei due segmenti con il centro di proiezione. Le corde che uniscono l'epicentro con i due punti di intersezione $F$ ed $F^{\prime}$ rappresentano le traccie dei due possibili piani di frattura e ci consentono di misurare gli angoli che i piani stessi formano con il raggio terrestre per l'epicentro. Dalla figura si sono ottenuti gli angoli di $66^{\circ}$ e di $16^{\circ}$.

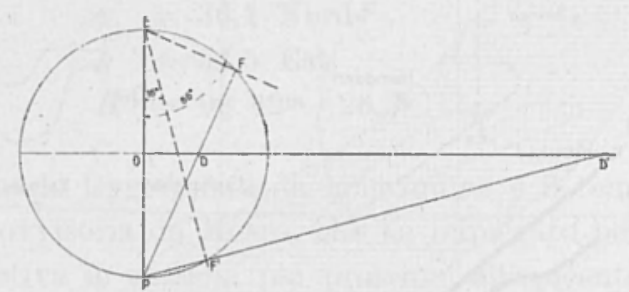

Fig. 2

Resta ora da decidere quale dei due piani possibili sia effettivamente il piano di frattura. Come è noto, a questo riguardo è necessario l'ausilio o di fatti precedentemente accertati o la conoscenza della geologia e della tettonica della zona ove è avvenuto il terremoto.

Nella fig. 3 abbiamo riportato schematicamente la carta della regione nord-occidentale dell'Iran dal punto di vista dell'orogenesi, secondo le indicazioni contenute nel trattato "L'evolution de la lithosphère " di Termier; abbiamo inoltre riportata su essa la posizione dell'epicentro. In base a tale carta si nota che le catene di monti che recingono la parte meridionale dello scudo iraniano (di origine prepaleozoica) sono per la massima parte formate da una successiva copertura di Altaidi che pur elaborate in epoche precedenti, si sono sovrapposte alla fine del secondario se non nel terziario. La catena dell'Alwand che è compresa tra Hamadan e Kermanshah è invece formata in prevalenza da rocce metamorfiche del paleozoico e quindi depositate in epoca precedente alla co- 
pertura delle Altaidi. L'epicentro cade nella zona di separazione tra le due formazioni e se si adotta per piano di frattura quello che forma con il meridiano un angolo di $126^{\circ}$ si nota che tale piano risulta praticamente parallelo non solo alla linea di demarcazione tra le due formazioni ma anche all'andamento delle catene montuose di origine recente che recingono il Golfo Persico.

Riteniamo quindi ragionevole pensare che la natura fisica della scossa che ha determinato il grrande terremoto del 13 Dicembre 1957 sia uno scorrimento tra due formazioni geologicamente di epoche di-

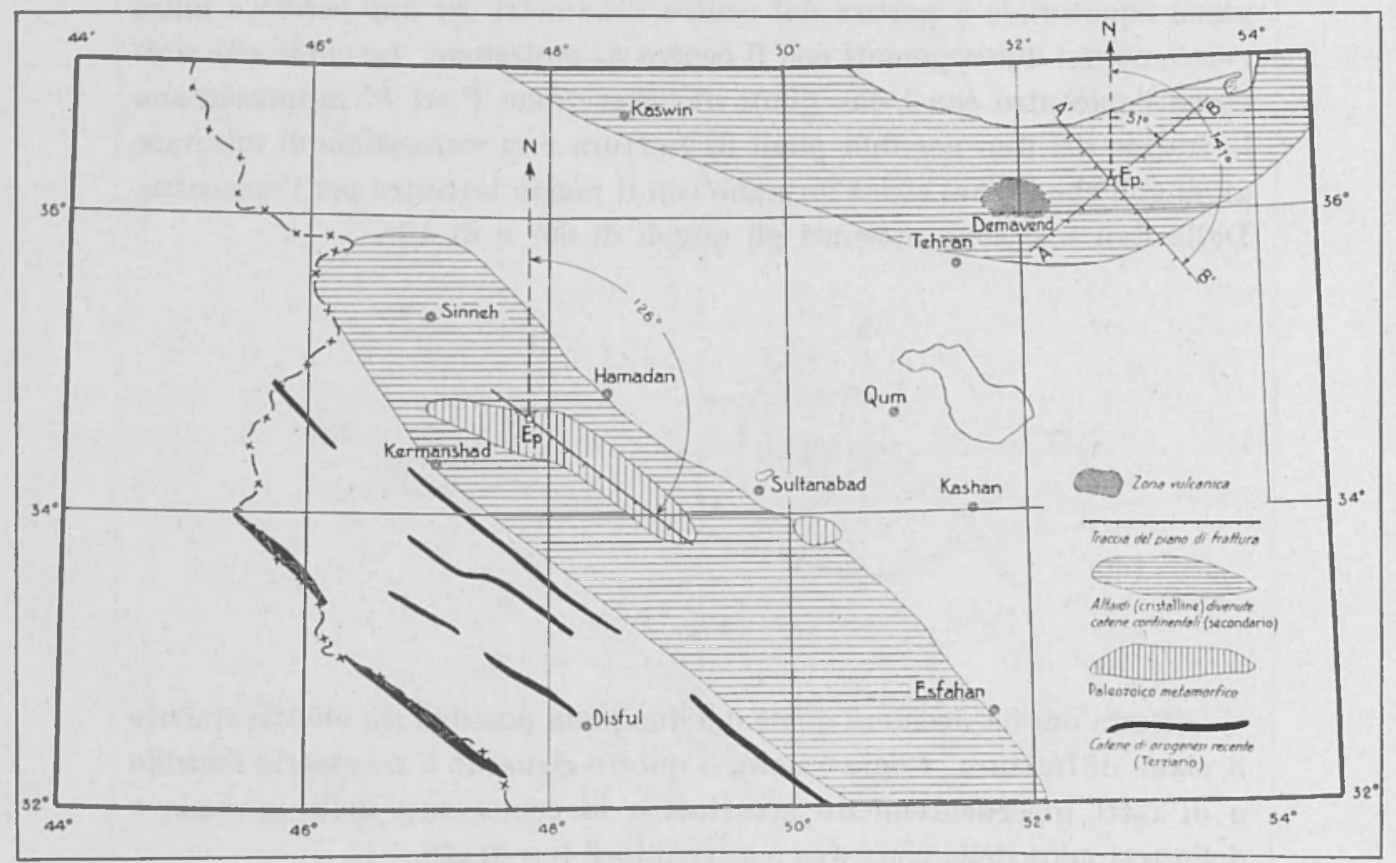

Fig. 3

verse, e che il piano di frattura sia quello che forma con il meridiano per l'epicentro un angolo di cn'ca $126^{\circ}$, e con il raggio terrestre passante per l'epicentro un angolo di $66^{\circ}$ circa. Se si tiene presente il fatto che la zona è già stata sede anche negli anni precedenti di altri movimenti tellurici, l'ipotesi di una orogenesi ancora in atto non è da escludersi.

Ir, TERREMOTO DEL 2 LUGLIO 1957.

Alle ore $0042^{\mathrm{m}}$ c. del 2 luglio 1957 sulla regione montana a Nord di Teheran si è verificato un violento terremoto avvertito almeno di II ${ }^{\circ}-I^{\circ}{ }^{\circ}$ 
anche nella capitale, l'area macrosismica molto estesa ha interessato qualche centinaio di villaggi molti dei quali hanno subito danni ingenti. La magnitudo del terremoto stesso ha raggiunto il valore di 7,4 e quindi il movimento tellurico è da classificarsi tra i più intensi avvenuti in Iran in questo ultimo cinquantennio. Numerose anche in questo caso sono state le repliche, tutte di minore entità, come si può riscontrare dall'esame dei bollettini dell'U.R.S.S.

La determinazione dei dati microsismici relativi alla posizione dell'ipocentro e il tempo origine è stata da noi eseguita utilizzando le registrazioni delle 20 stazioni riportate nella Tabella II, le cui distanze dall'epicentro sono comprese tra 800 e $2800 \mathrm{Km}$ e distribuite nel modo più uniforme possibile intorno all'epicentro stesso. Il metodo applicato è quello seguito per la determinazione dei dati ipocentrali del terremoto di Farsinaj per cui rimando direttamente alla pubblicazione a riguardo.

Siamo partiti dai seguenti dati provvisori:

$$
\begin{aligned}
& p=36,1 \text { Nord } \\
& \lambda=52,5 \text { Est } \\
& H=0042^{\mathrm{m}} 26,5^{\mathrm{s}}
\end{aligned}
$$

ottenuti modificando leggermente la longitudine e il tempo origine calcolati per via provvisoria da Mosca, che ha impiegato per la determinazione approssimativa le stazioni più prossime all'epicentro. Per quanto riguarda la profondità abbiamo assunto $h=33 \mathrm{Km}$ dato che in base alle registrazioni, il terremoto ha le caratteristiche di un terremoto a profondità normale.

La prima approssimazione ci ha fornito le seguenti correzioni da apportare ai dati di partenza.

$$
\begin{array}{ll}
\delta \varphi_{o}=+0^{\circ}, 1173323 & \delta \lambda_{o}=+0^{\circ}, 218567 \\
\delta h_{o}=+0,13299 & \delta t_{o}=+0^{\mathrm{s}}, 74533
\end{array}
$$

ove l'unità di misura per $\delta h_{o}$ è pari a 0,01 del raggio della Terra privata della crosta terrestre $(63,4 \mathrm{Km})$.

Con queste correzioni le coordinate più probabili dell'epicentro, a il tempo origine divengono

$$
\begin{aligned}
& \varphi_{o}=36^{\circ} 07^{\prime}, 04 \mathrm{~N} \\
& \lambda_{o}=52^{\circ} 43^{\prime}, 11 \mathrm{E} \\
& h=41,4 \mathrm{Km} . \\
& H=00^{\mathrm{h}} 43^{\mathrm{m}} 27^{\mathrm{s}, 2}
\end{aligned}
$$


Tabella II

\begin{tabular}{|c|c|c|c|c|c|c|c|c|}
\hline & \multirow{2}{*}{ Stazione } & \multirow{2}{*}{$\underset{\text { (gradi) }}{\Delta}$} & \multicolumn{2}{|c|}{ Tempo di } & \multirow{2}{*}{$\begin{array}{c}\text { Tempo } \\
\text { di origine }\end{array}$} & \multirow{2}{*}{ 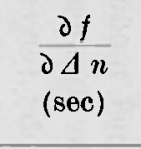 } & \multirow{2}{*}{$\frac{\partial f}{\partial h}$} & \multirow{2}{*}{$\begin{array}{r}T_{n}-\left[t_{o}+\right. \\
+f\left(\varphi_{o}, \lambda_{o}, h_{o}\right)\end{array}$} \\
\hline & & & Registraz. & $\begin{array}{c}\text { Tragitto } \\
\text { per } \mathrm{h}=33 \mathrm{~km}\end{array}$ & & & & \\
\hline 1 & Erevan & $07 \circ 20^{\prime}, 50$ & $00^{\mathrm{h}} 44^{\mathrm{m}} 18^{\mathrm{s}}, 4$ & $01 \mathrm{~m} 47 \mathrm{~s}, 6$ & $00^{\mathrm{h}} 42^{\mathrm{m}} 30^{\mathrm{s}}, 8$ & $+145,0$ & $-1 \mathrm{~s}, 2$ & $+4 s, 3$ \\
\hline 2 & Tiflis & $08 \quad 17,50$ & $\begin{array}{lll}44 & 26 & , 6\end{array}$ & 0200,8 & $42 \quad 25,8$ & $+13,9$ & $--1,6$ & $-0,7$ \\
\hline 3 & Bakuriani & 0903,15 & 4438,9 & 0211,4 & $42 \quad 27,5$ & $+13,8$ & $-1,9$ & $+1,0$ \\
\hline 4 & Borjom & $09 \quad 10,60$ & $44 \quad 41,3$ & 0213,0 & 4228,3 & $+13,8$ & $--1,9$ & $+1,8$ \\
\hline 5 & Piatigorst & 1050,00 & 4501,1 & 0231,3 & $\begin{array}{lll}42 & 29,9\end{array}$ & $+13,6$ & $-2,4$ & +3.4 \\
\hline 6 & Quetta & 1325,43 & 4535,0 & 0310,6 & 4224,4 & $+13,1$ & $-3,0$ & $-2,1$ \\
\hline 7 & Ksara & 1347,85 & 4543 & $03 \quad 15,4$ & $42 \quad 27,6$ & $+13,1$ & $-3,1$ & $+1,1$ \\
\hline 8 & Oburdam & $13 \quad 57,71$ & $45 \quad 40,4$ & $03 \quad 17,6$ & $42 \quad 22,8$ & $+13,1$ & $-3,1$ & $-3,7$ \\
\hline 9 & Gerusalem. & $14 \quad 56,75$ & 4601,0 & 0330,4 & 4230,6 & $+12,9$ & $-3,3$ & $+4,1$ \\
\hline 10 & Korog & 1521,63 & $45 \quad 57,7$ & 0335,8 & $\begin{array}{lll}42 & 21 & , 9\end{array}$ & $+12,8$ & $-3,4$ & $-4,6$ \\
\hline 11 & Warsak & $15 \quad 35,33$ & 4606 & 0338,8 & $42 \quad 27,2$ & $+12,8$ & $-3,5$ & $+0,7$ \\
\hline 12 & Karaki & $16 \quad 44,35$ & $46 \quad 19$ & 0353,4 & 4225,6 & $+\quad 12,5$ & $-3,7$ & $-0,9$ \\
\hline 13 & Mirzad & $17 \quad 15,22$ & 4624,6 & $03 \quad 59,9$ & $\begin{array}{lll}42 & 24 & , 7\end{array}$ & $+\quad 12,5$ & $-3,8$ & $-1,8$ \\
\hline 14 & Laore & 1840,70 & $46 \quad 40$ & $04 \quad 17,5$ & $42 \quad 22,5$ & $+10,6$ & $-4,7$ & $-4,0$ \\
\hline 15 & Istanbul & 1900,20 & 4646 & 0421,5 & 4224,5 & $+10,6$ & - 5,0 & $-2,0$ \\
\hline 16 & Dhera-Dun & 2204,91 & $47 \quad 18,0$ & $04 \quad 53,7$ & $42 \quad 24,3$ & $\begin{array}{r}+9 \\
+\quad 9\end{array}$ & $-5,7$ & $-2,2$ \\
\hline 17 & Atene & 2302,33 & 4731 & 0503,2 & $42 \quad 27,2$ & $\begin{array}{r}+7 \\
+\quad 9\end{array}$ & $-5,9$ & $\begin{array}{r}+\quad 0,7\end{array}$ \\
\hline 18 & Bombay & $24 \quad 47,42$ & $47 \quad 47,1$ & 0520,3 & $42 \quad 26,8$ & $\begin{array}{r}+4 \\
+\quad 9\end{array}$ & $-6,0$ & $+0,3$ \\
\hline 19 & Poona & $25 \quad 45,38$ & $47 \quad 57$ & 0529,3 & $42 \quad 27,7$ & $\begin{array}{r}+4 \\
+\quad 9.4\end{array}$ & $-6,1$ & $+1,2$ \\
\hline & Belgrado & 2548,31 & $\begin{array}{lll}47 & 59,0\end{array}$ & $\begin{array}{lll}05 & 29,8\end{array}$ & 4229,2 & $+\quad 9,4$ & $-6,1$ & $+2,7$ \\
\hline & & & & Media & $42 \quad 26,5$ & & & \\
\hline
\end{tabular}


CONTRIbUto alla CONOSCENza Delle Caratteristiche SiSmiche, eCC. 531

Siamo passati quindi alla seconda approssimazione i cui risultati sono stati i seguenti:

$$
\begin{array}{ll}
\delta \varphi_{o}=+0^{\circ}, 0534367 & \delta \lambda_{o}=+0^{\circ}, 0095157 \\
\delta h_{o}=-0,235679 & \delta t_{o}=-0^{\mathrm{s}}, 851355
\end{array}
$$

Quindi in base a questa approssimazione la profondità ipocentrale passa da $41,4 \mathrm{Km}$ a $26,4 \mathrm{Km}$ con un errore standard di $\pm 15 \mathrm{~km}$ ca. Questo ultimo, risultato da noi ritenuto non soddisfacente ci ha consigliata

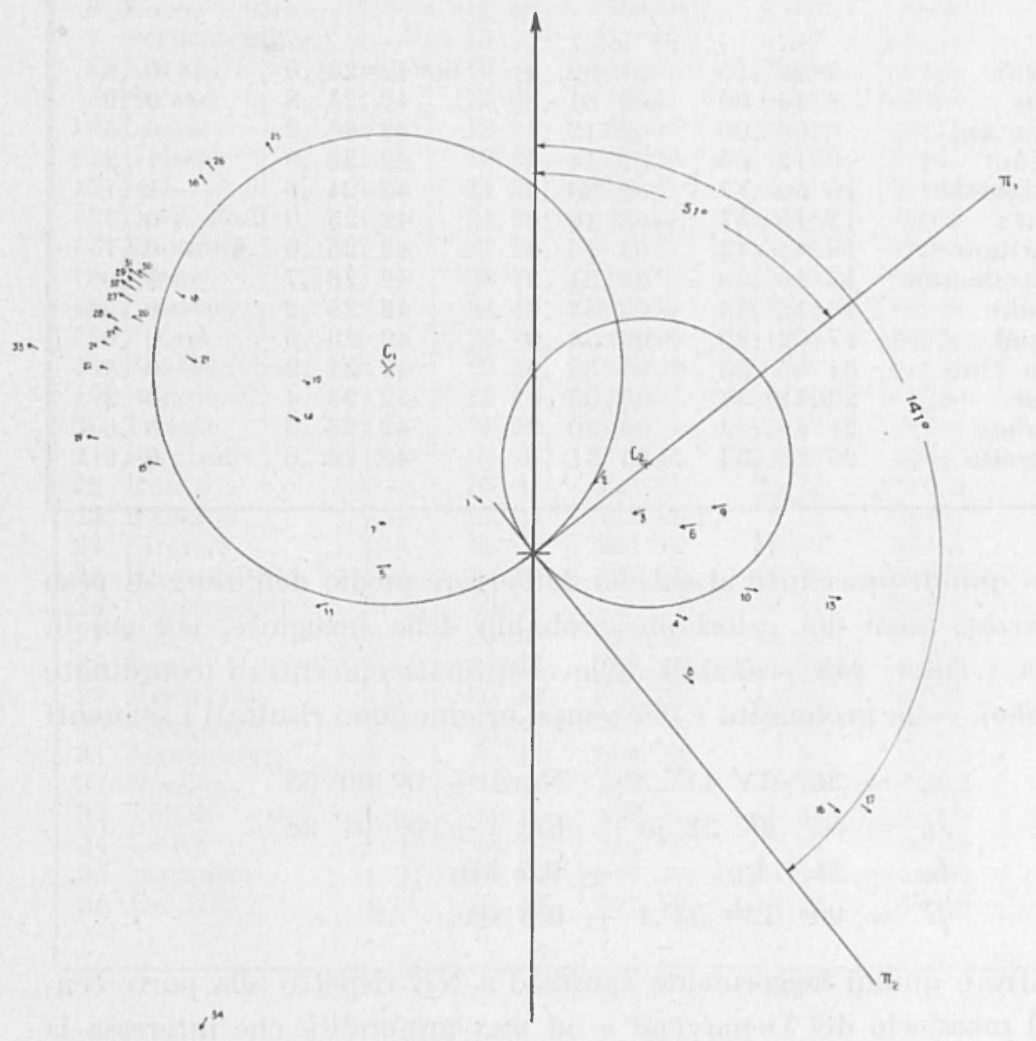

Fig. 4

l'esecuzione di una ulteriore approssimazione compiuta impiegando solo 14 stazioni, e cioè eliminando dai calcoli el stazioni di Poona, Ksara, Warsak, Laore, Is tanbul, Korog, che comportavano scarti superiori al secondo rispetto al tempo origine provvisorio. 
La tabella III riporta i dati necessari per quest'ultima approssimazione che ha condotto alle seguenti correzioni:

$$
\begin{array}{ll}
\delta \varphi_{o}=+0^{\circ}, 0082715 & \delta \lambda_{o}=+0^{\circ}, 0366099 \\
\delta h_{o}=-0,079396^{\circ} & \delta t_{o}=-0^{\mathrm{s}, 289722}
\end{array}
$$

\begin{tabular}{|c|c|c|c|c|c|}
\hline & \multirow[b]{2}{*}{ Stazione } & \multirow{2}{*}{$\stackrel{\Delta}{(\operatorname{grad} i)}$} & \multicolumn{2}{|c|}{ Tempo di } & \multirow{2}{*}{$\begin{array}{c}T_{n}-\left[t_{o}+\right. \\
\left.+f\left(\phi_{c}, \lambda_{o}, h_{o}\right)\right]\end{array}$} \\
\hline & & & $\begin{array}{c}\text { tragitto } \\
\mathrm{h}=26,4 \mathrm{~km}\end{array}$ & origine & \\
\hline & Erevan & $7 \circ 38^{\prime} .75$ & $01 \quad 52,4$ & $00^{\mathrm{h}} 42 \mathrm{~m} 26^{\mathrm{s}}, 0$ & $-0,8$ \\
\hline & Tiflis & $8 \quad 19,00$ & 0201,8 & $\begin{array}{lll}42 & 24,8\end{array}$ & $-0,9$ \\
\hline 3 & Bakuriani & 906,00 & $02 \quad 12,7$ & $42 \quad 26,2$ & $+0,5$ \\
\hline 4 & Borjom & 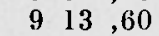 & $02 \quad 14,4$ & $\begin{array}{lll}42 & 26 & 9\end{array}$ & $+1,2$ \\
\hline & Piatigotsk & 1050,17 & $02 \quad 36,5$ & $42 \quad 24,6$ & $-1,1$ \\
\hline 6 & Quetta & $13 \quad 19,57$ & $03 \quad 10,0$ & 4225,0 & $-0,7$ \\
\hline 7 & Oburdam & 1344.43 & $03 \quad 15,4$ & 4225,0 & -0.7 \\
\hline 8 & Gerusalemme & $15 \quad 10,50$ & $03 \quad 34,3$ & $\begin{array}{ll}42 & 26,7\end{array}$ & $+1,0$ \\
\hline 9 & Karaki & $\begin{array}{l}16 \quad 42,38 \\
\end{array}$ & $03 \quad 53,8$ & $42 \quad 25,2$ & $-0,5$ \\
\hline 10 & Mirzad & 1702,25 & 0358,0 & $42 \quad 26,6$ & $+0,9$ \\
\hline 11 & Dera Dun & 2155,55 & 0453,1 & $\begin{array}{ll}42 & 24,9\end{array}$ & $-0,8$ \\
\hline 12 & Atene & $23 \quad 11,36$ & $\begin{array}{ll}05 & 05,6\end{array}$ & $42 \quad 25,4$ & $-0,3$ \\
\hline 13 & Bombay & $24 \quad 45,42$ & $\begin{array}{lll}05 & 20,7\end{array}$ & $42 \quad 26,3$ & $+0,6$ \\
\hline 14 & Belgrado & $\begin{array}{lll}25 & 53,33\end{array}$ & 0531,4 & $42 \quad 26,6$ & $+0,9$ \\
\hline
\end{tabular}

Tabella III

Abbiamo quindi proceduto al calcolo dell'errore medio dell'unità di peso e degli errori medi dei valori più probabili delle incognite, per cui in definitiva i valori più probabili delle coordinate epicentrali (coordinate geografiche), della profondità e del tempo origine sono risultati i seguenti

$$
\begin{aligned}
& \varphi_{0}=36^{\circ} 11^{\prime} 11^{\prime \prime}, 2 \quad \text { Nord } \pm 0^{\circ} 00^{\prime} 53^{\prime \prime} \\
& \lambda_{0}=52^{\circ} 46^{\prime} 22^{\prime \prime}, 3 \quad \text { Est } \pm 0^{\circ} 01^{\prime} 28^{\prime \prime} \\
& h_{0}=21,4 \mathrm{~km} \quad \pm 6,5 \mathrm{~km} \\
& H=00^{\mathrm{h}} 42^{\mathrm{m}} 54^{\mathrm{s}}, 4 \pm 0,5 \text { sec. }
\end{aligned}
$$

L'epicentro è quindi leggermente spostato a NE rispetto alla parte centrale del massiccio del Demavend e ad una profondità che interessa la zona inferiore dello strato del granito.

Anche per questo terremoto abbiamo voluto eseguire un tentativo per determinare la natura fisica della causa che ha originato il movimento sismico; a questo riguardo abbiamo impiegato gli impulsi iniziali delle registrazioni delle stazioni riportate nella Tabella IV, nella quale sono contenuti i dati necessari per la rappresentazione cartografica in proiezione stereografica polare con epicentro coincidente con l'ipocentro 
Tabella IV

\begin{tabular}{|c|c|c|c|c|c|c|}
\hline Stazioni & 嵒离 & $\begin{array}{c}\Delta \\
\text { (gradi) }\end{array}$ & $\begin{array}{c}\alpha \\
\text { (gradi) }\end{array}$ & $\begin{array}{c}v_{n} \\
(\mathrm{~km} / \mathrm{sec})\end{array}$ & $\cos e$ & $\operatorname{tang} \frac{1}{2} \Lambda^{\prime}$ \\
\hline $\begin{aligned} 1 & \text { Tiflis } \\
2 & \text { Piatigorsk } \\
3 & \text { Samarcanda } \\
4 & \text { Quetta } \\
5 & \text { Ksara } \\
6 & \text { Koros } \\
7 & \text { Gerusalemme } \\
8 & \text { Karaki } \\
9 & \text { Mirzad } \\
10 & \text { Laore } \\
11 & \text { Helwan } \\
12 & \text { Iasi } \\
13 & \text { Dera Dun } \\
14 & \text { Bucarest } \\
15 & \text { Atene } \\
16 & \text { Bombay } \\
17 & \text { Poona } \\
18 & \text { Bratislavua } \\
19 & \text { Reggio C. } \\
20 & \text { Trieste } \\
21 & \text { Belgrado } \\
22 & \text { Roma } \\
23 & \text { Bologna } \\
24 & \text { Firenze } \\
25 & \text { Uppsala } \\
26 & \text { Copenaghen } \\
27 & \text { Coira } \\
28 & \text { Pavia } \\
29 & \text { Mestetten } \\
30 & \text { Stoccarda } \\
31 & \text { Strasburgo } \\
32 & \text { Basilea } \\
33 & \text { Toledo } \\
34 & \text { Lwiro } \\
35 & \text { Matzuiro } \\
36 & \text { De Bilt }\end{aligned}$ & $\begin{array}{l}\mathrm{e}- \\
\mathrm{i}- \\
\mathrm{i}- \\
\mathrm{i}+ \\
\mathrm{i}- \\
\mathrm{i}- \\
\mathrm{i}- \\
\mathrm{i}+ \\
\mathrm{i}- \\
\mathrm{e}+ \\
\mathrm{i}+ \\
\mathrm{i}- \\
\mathrm{i}+ \\
\mathrm{i}- \\
\mathrm{i}+ \\
\mathrm{i}+ \\
\mathrm{e}+ \\
\mathrm{e}- \\
\mathrm{i}- \\
\mathrm{i}+ \\
\mathrm{i}- \\
\mathrm{i}+ \\
\mathrm{i}+ \\
\mathrm{i}+ \\
\mathrm{i}+ \\
\mathrm{i}+ \\
\mathrm{i}+ \\
\mathrm{i}+ \\
\mathrm{i}+ \\
\mathrm{i}+ \\
\mathrm{i}+ \\
\mathrm{i}+ \\
\mathrm{i}+ \\
\mathrm{i}+ \\
\mathrm{i}+ \\
\mathrm{i}+\end{array}$ & $\begin{array}{ll}80 & 19 \\
10 & 50 \\
11 & 49 \\
13 & 20 \\
14 & 01 \\
15 & 09 \\
15 & 10 \\
16 & 42 \\
17 & 02 \\
18 & 31 \\
18 & 39 \\
21 & 28 \\
21 & 55 \\
21 & 35 \\
23 & 11 \\
24 & 45 \\
25 & 39 \\
28 & 30 \\
29 & 11 \\
30 & 25 \\
25 & 53 \\
31 & 29 \\
32 & 04 \\
32 & 08 \\
32 & 25 \\
33 & 20 \\
33 & 25 \\
33 & 37 \\
33 & 54 \\
33 & 48 \\
34 & 43 \\
34 & 47 \\
44 & 11 \\
44 & 19 \\
66 & 25 \\
36 & 43\end{array}$ & $\begin{array}{rl}314025 \\
40 & 06 \\
68 & 19 \\
112 & 43 \\
265 & 17 \\
79 & 04 \\
281 & 46 \\
128 & 40 \\
75 & 57 \\
98 & 09 \\
256 & 29 \\
307 & 41 \\
98 & 14 \\
300 & 41 \\
283 & 13 \\
129 & 04 \\
127 & 35 \\
306 & 02 \\
285 & 07 \\
300 & 18 \\
299 & 30 \\
293 & 52 \\
297 & 55 \\
296 & 34 \\
327 & 46 \\
318 & 19 \\
302 & 10 \\
299 & 10 \\
304 & 33 \\
305 & 38 \\
305 & 08 \\
303 & 19 \\
292 & 31 \\
214 & 55 \\
61 & 10 \\
320 & 53\end{array}$ & $\begin{array}{r}8,00 \\
8,10 \\
8,22 \\
8,46 \\
8,48 \\
8,58 \\
8,59 \\
8,80 \\
8,90 \\
9,02 \\
9,02 \\
10,07 \\
10,09 \\
10,08 \\
11,40 \\
11,44 \\
11,78 \\
12,40 \\
12,42 \\
12,47 \\
11,80 \\
12,61 \\
12,70 \\
12,70 \\
12,75 \\
12,88 \\
12,89 \\
12,90 \\
12,92 \\
12,91 \\
12,93 \\
12,93 \\
13,87 \\
13,87 \\
17,34 \\
13,21\end{array}$ & $\begin{array}{l}97125 \\
95925 \\
94525 \\
91843 \\
91627 \\
90559 \\
90454 \\
88295 \\
87303 \\
86141 \\
86141 \\
77159 \\
77006 \\
77083 \\
68157 \\
67919 \\
65959 \\
62611 \\
62560 \\
62309 \\
65847 \\
61618 \\
61181 \\
61181 \\
60941 \\
60326 \\
60279 \\
60232 \\
60139 \\
60185 \\
60092 \\
60092 \\
56020 \\
56020 \\
44809 \\
58819\end{array}$ & $\begin{array}{l}0, \mathbf{2 4 5} \\
0,295 \\
0,345 \\
0,431 \\
0,437 \\
0,368 \\
0,471 \\
0,532 \\
0,559 \\
0,589 \\
0,589 \\
0,824 \\
0,828 \\
0,826 \\
1,073 \\
1,080 \\
1,140 \\
1,244 \\
1,247 \\
1,256 \\
1,143 \\
1,279 \\
1,293 \\
1,293 \\
1,309 \\
1,322 \\
1,324 \\
1,325 \\
1,329 \\
1,327 \\
1,330 \\
1,330 \\
1,479 \\
1,479 \\
1,996 \\
1,374\end{array}$ \\
\hline
\end{tabular}

e piano di proiezione al piano equatoriale corrispondente. Applicando il procedimento già eseguito per il terremoto precedente, e illustrato dalla fig. 4, abbiamo potuto stabilire che il movimento sismico è stato originato da una frattura; i due possibili piani di frattura previsti dalla teoria formano con il Nord geografico gli angoli di $51^{\circ}$ e di $141^{\circ}$ circa, mentre gli angoli di inclinazione dei piani stessi, rispetto al raggio terrestre passante per l'epicentro sono rispettivamente $\dot{\delta}_{1}=38^{\circ}, \beta_{2}=52^{\circ}$. 


\section{RIASSUNTO}

Proseguendo nello studio delle caratteristiche sismiche del massiccio iraniano, si determinano $i$ dati ipocentrali e l'ora origine del terremoto del 2 luglio 1957 avvenuto a $N$ di Teheran. Si è quindi indagato sulla natura del movimento sismico giungendo alla conclusione che trattasi di una frattura; di essa sono stati calcolati gli elementi caratteristici. Analogo studio condotto per il terremoto di Farsinaj del 13 Dicembre 1957, ha mostrato che anche per esso trattasi di una frattura.

\section{$A B S T R A C T$}

Following up the study of the seismic features of the Iranian massif, the hypocentral data and time of the origin of the earthquake which took place north of Teheran on July 2 1957, were worked out. Then the nature of this seismic movement was inquired into, reaching the conclusion that a fracture was involved. Salient features of this fracture were worked out. A similar study carried our in the case of Farsinaj earthquake of December 13 1957, demonstrated that it was also due to a fratucre.

\section{BIBLIOGRAFIA}

Peronaci F., Sicmicità dell'Iran, "Annuali di Geofisica ", XI, 1, (1958).

BYERLY P., The nature of the first motion in the Chilean earthquake of November 11, 1922, "America, Journ. Science ", XVI, 93, (1928).

BYERLY, P., The earthquake of July 6, 1934. Amplitudes and first motion "Bull. Seis. Soc. A mer. ", 28, (1938).

Di FilipPo, D., Sulla rappresentazione in superficie della natura dinamica di una scossa all'ipocentro, "Annali di Geofisica", III, 2, (1950).

Caloi, P., Peronaci, F., Il terremoto del Turkestan, "Annali di Geofisica", I, 2, (1948).

Termier, H.-Termier, G., L'évolution de la lithosphère, Paris 1957.

Furon, R., Géologie du Plateau Iranien, Mémoire du Muséum National d'IIistoire Naturelle, Paris, 1941. 